\title{
FUNDAMENTOS DEL DERECHO Y FUNDAMENTOS DEL CONOCIMIENTO DEL DERECHO*
}

\author{
RICARDO ALBERTO CARACCIOLO \\ Consejo Nacional de Investigaciones \\ Técnicas y Científicas, \\ Argentina
}

1. Una de las cuestiones que constituyen actualmente una fuente de controversia importante en la teoría de la ciencia se refiere a los fundamentos del conocimiento científico. Esta controversia, que se enmarca dentro de la crisis de los fundamentos de determinadas disciplinas consideradas hasta entonces paradigmas de conocimiento seguro, tiene que tener efectos inmediatos en las ciencias sociales y presumiblemente, en la medida de su reclamo de cientificidad, también en el conocimiento jurídico. Con todo, parecería que los teóricos del derecho no han tomado aún suficiente conciencia de las eventuales implicaciones que tal discusión puede tener en relación a su disciplina. Me propongo efectuar un análisis de una manera de pensar típica en la ciencia jurídica que puede contribuir a bloquear el paso a las conclusiones de esa polémica. Pienso que se puede sostener, también, que ese pensamiento es producto de una confusión.

2. Como es sabido, el esquema en el que se basa la interpretación clásica del conocimiento, en especial del conocimiento científico, consiste en suponer que todo saber racional tiene que tener fundamentos suficientes, esto es, tiene que ser posible indicar pruebas concluyentes en su favor. ${ }^{1}$ Fundamentar un conocimiento, entonces, significa proporcionar razones que aseguren la certidumbre de su verdad. Este esquema,

- Este trabajo fue presentado en la reunión académica de la Sociedad Argentina de Análisis Filosófico del 25 de junio de 1977.

1 Cf. Imre Lakatos: "Falsification and the Methodology of Scientific Programmes" en Criticism, and the Growth of Knowledge, editado por I. Lakatos y A. Masgrave: Cambridge, 1970, p. 91 y ss. 
que resulta de la aceptación del principio de razón suficiente como principio metodológico regular de la racionalidad del conocimiento, se proyecta al mismo tiempo como una exigencia de seguridad. Esto es, un enunciado cognoscitivo que tenga fundamentos suficientes no puede ser objeto de cuestionamientos ulteriores. ${ }^{2}$ De aquí, el concepto clásico de ciencia: una teoría aparece como un conjunto de enunciados que constituyen un sistema de relaciones lógicas establecidas a partir de un subconjunto de enunciados fundamentales. Por consiguiente, en tanto la relación de implicación asegura la transmisión de la verdad, el problema central del conocimiento consiste en la determinación de la verdad de los enunciados que tengan que ser admitidos como los "fundamentos". ${ }^{8} \mathrm{La}$ experiencia sensible o la intuición racional constituyen las alternativas que la tradición filosófica instituyó como fuentes privilegiadas del conocimiento de los fundamentos.

Este modelo de racionalidad extiende de hecho sus posibilidades más allá de las convicciones puramente cognoscitivas. El ámbito de lo normativo, el ámbito de los patrones de conducta que tengan que ser admitidos, puede ser juzgado también a partir de la exigencia de fundamentos seguros, a partir de la exigencia de que no pueden ser cuestionados.

Existen severas objeciones en contra de la pretensión de que pueda alcanzarse la certidumbre última en el conocimiento. En todo caso, aquéllas tienen que ver con el presente fracaso de las tentativas de tomar en cuenta conocimientos autofundamentados. A la par con el progreso científico que ha rebasado determinadas verdades consideradas clásicamente autoevidentes mediante el solo recurso de la razón, el empirismo a ultranza no puede evitar que la base empírica de la ciencia presuponga un instrumento racional de interpretación a fin de asegurar la equivalencia de las percepciones sensi-

2 Sobre la exigencia de certidumbre en el esquema clásico de interpretación de la ciencia, cf. Hans Albert: Teoría crítica, Buenos Aires, 1973, p. 20 y 89.

s Cf. para el concepto de teoría crítica y sus modificaciones, Friedrich Kambartel: Experiencia y estructura, Buenos Aires, 1973, p. 59 y 8 . 
bles. ${ }^{4}$ La teoría de la ciencia actual, por lo tanto, discute las alternativas posibles al modelo clásico del conocimiento. ${ }^{5}$ No es mi intención incursionar aquí en la polémica que las mismas suscitan. Pero, en todo caso, las alternativas muestran, a esta altura, que no parece razonable identificar el dominio de lo "racional" con aquello susceptible de ser representado según los moldes de lo que Lakatos denomina "justificacionismo"."

3. Me interesa, en cambio, la manera como se presenta la cuestión de los fundamentos en relación al conocimiento jurídico. ¿Es posible trasladar la polémica relativa a las objeciones a la interpretación clásica de la ciencia al dominio de la ciencia jurídica? ¿Existen en esa ciencia determinados enunciados o formulaciones teóricas que tengan que ser consideradas como sus "fundamentos"? De ser así, ¿de qué manera se ha intentado justificar la verdad de esos primeros enunciados?

Estas preguntas conducen, de alguna manera, a un modo peculiar de pensamiento que tiene que ser previamente elucidado en sus alcances si se quieren obtener respuestas relevantes. En verdad, sin un análisis de esa manera de pensar que resalte sus deficiencias, es posible que el planteamiento de esas preguntas carezca de sentido para los juristas. Existen profundas diferencias entre los teóricos que se han ocupado del conocimiento jurídico. Sin embargo, es admisible indicar dos características generales compartidas que, en conjunto, definen una forma de enfocar el problema de los fundamentos en la ciencia jurídica.

a) En primer lugar, hay que indicar el importante papel

- Lakatos, ob. cit., p. 94 y ss.: Kambartel, ob. cit., p. 239.

- En especial, pienso que deben ser destacadas las tesis de Karl Popper y Thomas Khun. El libro citado Criticism and the Growth of Knowledge constituye una excelente muestra del nivel alcanzado por la confrontación de los puntos de vista de ambos autores. También, la teoría de la ciencia de Popper es discutida por epistemólogos, de raíz hegeliana, en La disputa del positivismo lógico en la sociología alemana, editado por Theodor Adorno, Barcelona, 1972.

- Lakatos, ob. cit., p. 93. 
que se le asigna al modelo clásico de fundamentación racional en la ciencia jurídica, papel que se traduce en las elaboraciones relativas al concepto de "sistema".?

b) En segundo lugar, tales formulaciones no se refieren, o por lo menos no primordialmente, a una fundamentación de enunciados teóricos a pesar de la relevancia epistemológica que expresamente se les reconoce; el problema de la fundamentación teórica se confude aquí con el problema de la fundamentación normativa.

En efecto, en la ciencia del derecho, la cuestión de los fundamentos del conocimiento y la idea del sistema que la acompaña se disuelven tradicionalmente en el problema de los fundamentos de las normas, que vendrían a constituir el material de conocimiento jurídico. De esta forma, el objetivo principal del modelo clásico de la ciencia, a saber, la tentativa de proporcionar las bases de certidumbre última del conocimiento, se transfiere al dominio del conocimiento jurídico como una cuestión relativa a la justificación racionalmente fundamentada de las directivas de conducta, las que, prima facie, vendrían a constituir el "derecho" como objeto de la ciencia jurídica. Esto es, aún en análisis recientes de metología jurídica, la tarea de la ciencia del derecho vendría a consistir, en lo que a búsqueda de fundamento se refiere, en la determinación de los postulados normativos a tenor de los cuales se estructura sistemáticamente el material del conocimiento jurídico. En la extrapolación del modelo "justificacionista", la explicación científica a partir de la teoría asumida como fundamento, se convierte en última instancia en "justificación normativa" de decisiones a partir del sistema aceptado de normas.

7 Tesis doctoral del autor, El derecho como sistema. Análisis de un esquema conceptual, presentada en la Universidad Nacional de Córdoba, Argentina.

${ }_{8}$ Cf. Carlos Alchourrón y Eugenio Bulygin: Normative Systems, Nueva York, 1971, p. 168 y ss. Aunque el contexto en el cual los autores se refieren a la noción de "justificación normativa" no presupone la admisión de la tesis de que pueda hablarse de "fundamentos últimos del derecho", o de "normas fundamentales", la misma es relevante aquí por lo siguiente: en cuanto significa reemplazar la exigencia de que tengan que fundarse los enunciados 
Ambas operaciones tendrían fundamentalmente la misma estructura lógica. Explícitamente se reconoce aquí lo siguiente: la ciencia del derecho no tendría por función explicar realidad alguna, sino, más bien, suministrar pautas de justificación de decisiones sobre patrones de comportamiento. A pesar de ello, se insiste en el carácter cognoscitivo de la ciencia jurídica. Me propongo analizar los problemas que presenta esta manera de pensar.

4. Es fácil advertir que la extrapolación en cuestión, responde a la tentativa de trasladar los patrones de racionalidad que el modelo supone al conjunto de las normas que se admiten como "derecho". Así, en definitiva, esta racionalidad es presentada como una exigencia epistemológica: los juristas no estarían en condiciones de suministrar conocimientos fundados acerca de normas si no fuera posible reconstruirlas sistemáticamente como conjuntos consistentes de expresiones lingüísticas. ${ }^{\circ}$ Por consiguiente, en esta perspectiva, pareciera que la búsqueda de los fundamentos normativos conduce, al mismo tiempo, a la fundamentación paralela del conocimientri acerca de normas: los enunciados teóricos no podrían fundamentarse a menos que se identifique, en el mismo acto de conocimiento, las premisas que vengan a constituir las bases del sistema normativo propuesto como objeto de conocimiento, es decir, si no se muestra que las normas del conjunto se justifican racionalmente de alguna manera. Esto es claro, por ejemplo, en la elaboración kelseniana de la noción de "norma fundamental": esta aparece simultánea y ambigua-

teóricos de la disciplina jurídica, por la exigencia de que tengan que justificarse normativamente. Por consiguiente, cabe también preguntarse acerca del sentido que puede tener la afirmación de que una ciencia, un conjunto de enunciados con pretensión cognoscitiva tenga que recurrir a "justificaciones normativas". No hay duda que el analisis que sigue es aplicable en gran medida a la concepción de la ciencia jurídica de Alchourrón y Bulygin. Por otra parte, la identificación de los problemas de fundamentación no implica asignarle al jurista la función de justificar normas. Pero sí se supone que otorgar prioridad, como trataré de mostrar, a la cuestión de la fundamentación normativa como condición del conocimiento.

9 Cf. Hans Kelsen: Contribuciones a la teoría pura del derecho, Buenos Aires, 1969 , p. 98. 
mente como punto de partida de las relaciones de validez normativa y como el conjunto de enunciados teóricos de la ciencia del derecho. ${ }^{10}$

Cabe interpretar esta identificación de los problemas de fundamentación por lo menos de dos maneras diferentes, cada una de las cuales introduce sus propias complicaciones en el plano de la metateoría de la ciencia jurídica.

5. Según la primera posibilidad, esta identificación es la consecuencia de una identidad genuina de los fundamentos del derecho como conjunto de normas y de la ciencia del derecho como conjunto de enunciados. Ahora bien, ¿de qué manera puede ser esto posible? A menos de que se asimilen ambas categorías semánticas, se trata aquí o bien de normas "fundamentales" o bien de enunciados "fundamentales"; alternativas que voy a denominar I y II.

En la alternativa I hay que sostener la discutible tesis según la cual es posible inferir la "verdad" de enunciados teóricos a partir de premisas normativas," cuyo "status ontológico" no puede ser explicado, obviamente, por la propia teoría que se fundamenta en las mismas. De lo contrario, lo cual resulta más plausible, hay que admitir, en cuanto se sostenga esa fundamentación, que las formulaciones de los juristas son en sí mismas formulaciones normativas y no enunciados teóricos. Así, se elimina el problema de los fundamentos del conocimiento jurídico: los juristas contribuyen con su labor intelectual al proceso de creación normativa, cualesquiera que sean las diferencias que se postulen en cuanto al grado de vigencia social de esas formulaciones en su relación con aquéllas de los órganos "oficiales" de creación. De aquí, puede decirse que la ciencia del derecho "... no

10 Cf. Roberto Vernengo: La naturaleza del conocimiento juridico, Buenos Aires, 1973, p. 149 y ss.

11 Para el estado actual de este problema: Norbert Hoerster: Problemas de ética normativa, Buenos Aires, 1975, pp. 9-39. 
está detrás o al lado del derecho sino que puede conformar el derecho mismo y la vida en y bajo el derecho". ${ }^{12}$

Y de aquí también, la asimilación de las tareas de interpretación de jueces y juristas: ambos justifican normativamente lo que afirman en el mismo sistema de normas. ${ }^{13} \mathrm{La}$ elección de esta alternativa explica asimismo el hecho de que, en la búsqueda de los fundamentos sistemáticos de la ciencia del derecho, la discusión entre racionalistas y empiristas se haya centrado en la posibilidad o imposibilidad de determinar mediante el solo recurso de la razón, la "validez" de las normas "fundamentales". ${ }^{14}$

De acuerdo a la alternativa II hay que admitir la tesis, problemática por razones lógicas y epistemológicas, de acuerdo con la cual es posible fundamentar la validez de enunciados normativos en premisas teóricas. Se presenta históricamente como un procedimiento típico, y no solo en la denominada "jurisprudencia de conceptos", que es la elaboración de un aparato conceptual teórico compuesto de definiciones que trascienden al lenguaje normativo, con el objeto de inferir del mismo pautas inéditas de conducta. ${ }^{15} \mathrm{Si}$ no se admite esta posibilidad de inferencia dentro de la alternativa II, entonces de la identidad de los "fundamentos" se sigue la eliminación del problema de los fundamentos del derecho, en cuanto a la cuestión relativa a las bases del conocimiento jurídico. De los enunciados teóricos "fundamentales" sólo es posible inferir enunciados y, por consiguiente, sólo es posible justificar actos de conocimiento, sin que tal posibilidad signifique abrir juicio de antemano acerca de la cuestión

12 Karl Engisch: Introducción al pensamiento jurídico, Madrid, 1967, p. 16. C. también la opinión de que la ciencia jurídica constituye una "ciencia práctica" en Karl Larenz: Metodología de la ciencia del derecho, Barcelona, 1966, pp. 19 y 21.

18 Alchourrón y Bulygin, ob. cit., p. 153 y s8.; p. 168 y 88.

14 Alchourrón y Bulygino ob. cit., pp. 51 y 53.

15 Cf. "La critica de Larenz al método constructivista" del primer Thering. Larenz, ob. cit., p. 39 y s6. No hay duda que la misma objeción básica puede ser dirigida a los planteos jusnaturalistas que pretenden inferir normas de la "naturaleza de las cosas", en última instancia, de enunciados descriptivos. Cf. Ernesto Garzón Valdez: Derecho y naturaleza de las cosas, Córdoba, 1971. 
teórica de saber si el modelo justificacionista entendido como modelo de inteligibilidad del derecho, es viable o no, para interpretar, no ya al conocimiento científico del derecho sino a la "realidad jurídica". En consecuencia, no hay inconveniente alguno en plantear con respecto al conjunto de enunciados de la ciencia jurídica, los mismos problemas que se derivan de la crisis del esquema clásico de interpretación de la ciencia. La presunta racionalidad del derecho no es, entonces, nada más que un producto de la racionalidad del conocimiento, de la misma manera que la racionalidad de la naturaleza o de los fenómenos sociales resulta epistemológicamente de los actos de conocimiento de las ciencias respectivas.

6. Según una segunda manera de interpretar la identificación de los problemas de fundamentación, esta no se sigue de la identidad de los "fundamentos" sino del paralelismo de los discursos teóricos y normativos respectivamente. A los enunciados "fundamentales" de la ciencia jurídica corresponden aquí, simétricamente, las normas "fundamentales" del derecho. ${ }^{18}$ Desde este punto de vista, la exigencia según la cual solo es posible fundamentar el conocimiento jurídico en la medida en que se identifiquen los fundamentos del derecho, parece conducir a la exigencia de que se establezcan relaciones de isomorfismo entre las estructuras de ambos niveles de lenguaje. La exigencia de isomorfismo entre el lenguaje de las normas y el lenguaje acerca de normas puede contribuir a explicar la tesis kelseniana que afirma que las contradicciones normativas se reflejan, o mejor, deben reflejarse, en contradicciones paralelas en el sistema de enunciados. En la medida en que un conjunto contradictorio de enunciados no puede considerarse, de manera alguna, como expresivo de un conocimiento fundamentado, esta exigencia concluye

16 Kelsen pretende de alguna manera distinguir la proposición o enunciado que describe la "norma fundamental" de la misma "norma fundamental", a pesar de que ambas son idénticas en su expresión lingüística. Cf. Hans Kelsen: Teoría pura del derecho, edición de circulación interna para el Instituto de Filosofía del Derecho de la UNBA, Buenos Aires, 1969, p. 124. 
en la afirmación de que es imposible el conocimiento de normas contradictorias. Esta segunda interpretación resulta de la aceptación en el nivel de la teoría jurídica de un modelo de inteligibilidad según el cual el "derecho" en tanto conjunto de normas, constituye un conjunto racional a tenor de los patrones clásicos de racionalidad del conocimiento. Es decir, aquí la teoría misma proporciona mediante su estructura el único paradigma válido para comprender el derecho.

Ahora bien, he tratado de mostrar en una investigación anterior, ${ }^{17}$ que este modelo del "derecho como sistema" supone comportamientos racionales de los individuos encargados del proceso creador de normas, es decir, que el derecho en tanto "realidad", producto de ese proceso, posee su propia "lógica", su propia estructura racional. De lo contrario, no existirian dos estructuras lingüísticas independientes entre las que se propone establecer relaciones de isomorfismo. Entonces, los criterios de fundamentación normativa no parecen depender de los actos cognoscitivos sino de los comportamientos racionales. ${ }^{18}$. Existen serias objeciones formulables en relación a este modelo teórico acerca del derecho. Últimamente, por ejemplo, Niklas Luhmann le imputa una subestimación del problema de la complejidad que el fenómeno social de la normación tiene que enfrentar, en cuanto reduce al "derecho" a las normas susceptibles de legitimación en aquellas propuestas consideradas como "fundamentales". ${ }^{10}$ Interesan aquí, sin embargo, los problemas que la aceptación de esta manera de concebir el derecho provoca en la cuestión de la fundamentación del conocimiento jurídico.

7. Pienso, en ese sentido, que se pueden indicar los siguientes problemas:

a) Esta manera de pensar parece corresponder, una vez admitida la distinción de niveles de lenguaje, a una con-

17 Cf. El derecho como sistema. Análisis de un esquema conceptual, ob. cit.

18 Para un análisis de los criterios sistemáticos, cf. Joseph $\mathrm{Ra}$, The Concept of Legal System, Oxford, 1971.

19 Cf. Niklas Luhmann: Ilustración sociológica y otros ensayos, Buenos Aires, 1973. p. 124. 
cepción según la cual la función de la ciencia del derecho consiste en describir "dogmáticamente" las normas consideradas válidas, descripción que supone su presentación en forma sistemática. ${ }^{20}$

Aquí, en tanto que resulta difícil admitir que un conjunto de puras descripciones puedan fundamentarse sistemáticamente, la unidad de la ciencia jurídica aparece como "reflejo" del carácter sistemático de la "realidad" jurídica. A pesar de la opinión de Kelsen de que sólo se puede hablar en sentido figurado de conexiones lógico-deductivas entre normas, en tanto y en cuanto los enunciados que las describen pueden presentarse, a su vez, en forma deductiva. ${ }^{21}$ La situación acerca de las relaciones entre normas y enunciados parece ser, justamente, la inversa, como señala Ross: la estructura deductiva de una serie de enunciados descriptivos de normas presupone la posibilidad de estructurar deductivamente las normas en cuestión. De donde resulta la imposibilidad de reducir la lógica deóntica a la lógica de los enunciados descriptivos. ${ }^{22}$ El problema de lograr una conexión sistemática que sirva para fundamentar los enunciados descriptivos de normas es todavía más difícil cuando se propone una vinculación normativa que no tiene paralelo en el nivel de los enunciados. Es lo que sucede cuando se trata de pensar al derecho según el "principio de legalidad". Así, cabe la pregunta acerca de la cuestión de saber en qué medida puede hablarse, en esta perspectiva, de fundamentación del conocimiento. La tarea de reconstruir el material normativo según los patrones propuestos, tiene una prioridad tal, que convierte en asunto secundario aquel problema.

b) Ahora bien, ¿cómo puede, desde el punto de vista epistemológico, justificarse con prioridad? En realidad, la exigencia de isomorfismo es muy fuerte cuando se trata del pro-

20 Que la ciencia jurídica sea descriptiva de normas, es una opinión común que justifica su denominación de "ciencia normativa". Cf. Jerzy Wroblewski, "Normativity of Legal Science", Logique et Analyse, 33, 1966, pp. 70-75.

21 Hans Kelsen: Teoria pura del derecho, ob. cit., p. 65.

22 Cf. Alf Ross: Directives and Norms, Londres, 1968, pp. 139-14. 
blema de fundamentación del conocimiento jurídico, a pesar de que constituye la base de la identificación de los problemas de fundamentación. En efecto, si denominamos $R_{1}$ a la relación de fundamentación de un conjunto de enunciados descriptivos de normas $E, E_{1}, E_{2} \ldots E_{n}$, y $R_{2}$ a la relación de fundamentación de un conjunto de normas $N, N_{1}, N_{2} \ldots N_{n}$, entonces la exigencia de isomorfía puede formularse de la manera siguiente:

$$
R_{1}\left(E, E_{1}, E_{2} \ldots E_{n}\right)=R_{2}\left(N, N_{1}, N_{2} \ldots N_{n}\right)
$$

Cuando esta correlación es presentada como exigencia epistemológica fundamental, es decir, como una condición de validación de conocimiento, es imposible cualquier reconstrucción racional de enunciados teóricos. Así, la reconstrucción metateórica de un conjunto $T$ de enunciados como expresivo de un conocimiento de un conjunto $T_{1}$ de normas; presupone como criterio de reconstrucción la propia estructura del conjunto $T_{1}$ que presumiblemente tiene que ser conocido: Pero, por hipótesis, conocer una "realidad", en este caso, conocer la estructura de un conjunto de normas significa tener la posibilidad de formular enunciados susceptibles de ser reconstruidos racionalmente. Por consiguiente, si se quiere mantener la posibilidad de conocimiento hay que admitir la independencia del problema de la fundamentación teórica. La estructura del conjunto normativo de la cual se puede hablar no es sino la estructura descrita por los enunciados, por lo que la fundamentación de éstos últimos no puede depender de lo que no puede ser conocido sino a través de descripciones. Salvo que se postule un acto de intelección especial, no discurre en relación al conocimiento jurídico.

En verdad, una eventual relación de similitud estructural no puede ser establecida en un tercer nivel de lenguaje $y$, en última instancia, mediante la construcción de sistemas axiomáticos en relación a los cuales los discursos teóricos y normativos puedan ser considerados modelos en sentido lógico. 
Que en todo caso tengan que elaborarse tales sistemas axiomáticos como premisa del conocimiento jurídico constituye, sin duda, una petición de principio que se explica en última instancia por una confusión de los niveles de lenguaje. Como ha sido demostrado por Carlos Alchourrón, una relación de isomorfismo entre el cálculo de normas y el cálculo de enunciados acerca de normas, sólo puede darse bajo dos condiciones, a saber, consistencia y completud. Que sólo son características contingentes y no necesarias de los conjuntos de enunciados y de los conjuntos de normas. ${ }^{23}$

c) La identificación de los problemas de fundamentación de acuerdo a esta segunda manera de interpretarlos parece corresponder también a una concepción sobre la relación entre teoría y realidad que actualmente es objeto de serias objeciones. Esta consiste en la suposición según la cual en la realidad operan las mismas estructuras lógicas que operan en la teoría, de manera tal, que le compete a la ciencia reproducir en forma completa esas relaciones estructurales que, en verdad, sólo parecen ser el producto de extrapolaciones previas. Es más, con frecuencia y en especial en el campo de las ciencias sociales, el conocimiento genuino es identificado con la posibilidad de reproducir teóricamente determinadas estructuras sin que interese aquí que estas estructuras tengan que ser consideradas las únicas "reales". ${ }^{4}$ Esta manera de concebir el conocimiento científico se manifiesta de inmediato en la exigencia de isomorfismo entre el conjunto de enunciados y la "realidad" jurídica. Y ciertamente, sea lo que sea la "realidad" jurídica, no existen pruebas de que puedan establecerse tales relaciones entre teoría y realidad.

En la medida en que se admite que toda ciencia se refiere a abstrac ciones que representan en forma aproximada características de la realidad, es claro que sólo se consideran ciertas variables entre las que se introducen teóricamente sólo

23 Cf. Carlos Alchourrón: "Logic of Norms and Logic of Normative Propositions", Logique et Analyse, 47, 1969, pp. 243-268.

24 Cf. B. Holzner: "Wholes in Social Sciences", Archiv für Rechs and So. cial Philosophie, XLIX/2, 1963, p. 137 y ss. 
algunas relaciones, sin que, por otra parte, la estructura sistemática de la teoría, como propiedad lógica y semántica, tenga que reproducir necesariamente una unidad objetivamente existente. ${ }^{25}$ En todo caso, la correspondencia estructural tiene que ser probada a menos que se la convierta en una cuestión metafísica. Esto último es lo que sucede con la metateoría de la ciencia jurídica que tiende a identificar los problemas de fundamentación.

8. Existe una manera de tratar de evitar la objeción precedente. Consiste en afirmar que el esquema de comprensión estructural del derecho es un presupuesto no dilucidable teóricamente. No hay inconveniente alguno en referirse aquí directamente a las tesis kelsenianas en cuanto constituyen la versión más acabada de esta manera de pensar.

La prioridad de la reconstrucción del material normativo en lo que se refiere a la validez del conocimiento jurídico en detrimento del problema de fundamentación teórica, puede ser sostenido de la manera siguiente: el modelo de fundamentación de las normas constituye un esquema epistemológico necesario de ordenación de un determinado material, para que el mismo pueda ser objeto de conocimiento jurídico, para que, entonces, se transforme en material juridico. Entonces el esquema en cuestión puede ser considerado como constitutivo, en el sentido kantiano, de toda posible experiencia que funcione como presupuesto del discurso teórico acerca del derecho. ${ }^{26}$ Esto equivale a afirmar que el derecho sólo puede ser conocido en tanto estructura peculiar de un conjunto de normas. $\mathrm{O}$ en lenguaje kelseniano: sólo es posible la "existencia" de normas en la medida en que los resultados de ciertos actos humanos puedan ser incluidos en una estructura definida por relaciones de fundamentación, puesto que sólo así esos resultados pueden ser pensados como "nor430.

25 Cf. Mario Bunge: La investigación científica, Barcelona, 1969, pp. 419 y

${ }_{28}$ Cf. Hans Kelsen: Teoría pura del derecho, ob. cit., p. 126. 
mas válidas". Puede entonces proponerse la siguiente versión simplificada del proceso de conocimiento jurídico:

a) material empírico indiscernible

b) material jurídico como resultado de la reconstrucción necesaria del material empírico

c) enunciados descriptivos del material identificado como "derecho"

d) reconstrucción de los enunciados descriptivos.

Si estos pasos son aceptables en principio, es clara la razón por la cual la tarea de fundamentación de la ciencia jurídica aparece como una cuestión secundaria: en tanto se la concibe como un conjunto de descripciones de una estructura normativa, basta respetar en la descripción la ordenación normativa para que tales enunciados no precisen de otra fundamentación.

En este caso, la reconstrucción, o mejor, la posibilidad de reconstrucción normativa funciona como un presupuesto de la realidad "descriptible". Tal presupuesto se manifiesta en la teoría kelseniana con la noción de "normas fundamentales". Ciertamente, la interpretación de la frase "si alguien manda y es obedecido, debe ser que mande y sea obedecido" con la que, en diversos contextos, Kelsen pretende expresar aquella noción, presenta graves dificultades. Sin embargo, parece claro que en tanto presupuesto de todo conocimiento acerca de normas, no puede al mismo tiempo ser pensada como "norma válida" en el mismo sentido en que lo son las normas que tiene que constituirse como tales. ${ }^{27}$ Esto es, no puede ser considerada como el "fundamento" del derecho. Más bien hay que considerarla según el propio objetivo kelseniano como un instrumento conformador de datos que sólo así se constituyen en objetos descriptibles. En definitiva, si se tiene en cuenta que la expresión "debe ser obedecido" da cuenta del carácter de "obligatoriedad" que Kelsen vincula a toda nor-

27 Cf. Vernengo, ob. cit., pp. 150-151. 
ma, se trata de una manera elíptica de definir los términos "normas" y "derechos". ${ }^{28} \mathrm{O}$ por lo menos, de establecer las condiciones necesarias de adecuación de una definición del concepto "norma" y nociones correlacionadas.

Ahora bien, ¿cuál es el status de un esquema conceptual de este tipo? Hay que advertir, que su postulada prioridad depende de su independencia de las formulaciones descriptivas de normas, esto es, que es el punto de partida previo y necesario de esas formulaciones. De otra manera, su justificación como esquema de interpretación de un determinado material, conduce de nuevo al problema de fundamentación de los enunciados de la ciencia jurídica. Los pasos $b$ y $c$ en el proceso de conocimiento tendrían, en este caso, que unificarse. Está claro, sin embargo, que, admitida esa prioridad, un esquema tal no puede ser confirmado ni refutado por ninguna experiencia, sea esta de la clase que sea, puesto que deliberadamente es presentado como la posibilidad de toda experiencia acerca de lo jurídico.

Pero, por otro lado, pareciera que tampoco hay medios de cuestionar su eventual incapacidad explicativa. Su presentación como categoría epistemológica necesaria apunta en última instancia, a una particular ontología de los “objetos" jurídicos, puesto que se trata de la única manera en que la "realidad" jurídica es cognoscible. Esto es, el esquema en cuestión es pensado como la única vía de acceso al conocimiento. ${ }^{29}$

Como, por otra parte, no resulta, como queda dicho, de formulaciones teóricas de los juristas, en la medida en que funciona como presupuesto del discurso científico, se sigue que la estructura peculiar del derecho así definida, es una condición necesaria para formular enunciados verdaderos acerca de normas $y$, por ende, para fundamentar esos enun-

28 Cf. Alchourrón y Bulygin, ob. cit., p. 73. Nota 2. Explícitamente se considera allí, a la "norma básica" como una regla conceptual, designado el uso del término "validez".

29 Que la teoría kantiana del conocimiento conduce a una ontología de los objetos de la experiencia, ha sido señalado por Kambarte, ob. cit., p. 145. 
ciados. Por consiguiente, la estructura de fundamentación normativa no es el resultado de descripciones ni de hipótesis teóricas formulables en el nivel de la ciencia. Más bien, no son concebibles descripciones o hipótesis acerca del derecho que no tengan en cuenta esa estructura. El mismo acto de conocimiento de normas pareciera implicar aquí la actividad mental que consiste en la reconstrucción de la estructura normativa a la que las normas pertenecen. Conocer el derecho equivale en cierto sentido a fundamentar normas. Así, entonces, el discurso científico del derecho no describe ni pretende describir estructuras de sistemas de normas sino que presupone una determinada estructura en sus descripciones. Este presupuesto es el único, en verdad, susceptible de justificar en la metateoría de la ciencia jurídica una exigencia de similitud estructural entre normas y enunciados y, por consiguiente, una identificación de los problemas de fundamentación.

9. Ahora bien, esta manera de pensar no es sino una versión racionalista del "justificacionismo" en el dominio del conocimiento jurídico, adaptado a la necesidad postulada de juzgar la racionalidad del derecho según los mismos criterios. Implica en definitiva la afirmación según la cual la racionalidad del conocimiento jurídico presupone la racionalidad del derecho. Por consiguiente, vale a ese respecto la misma objeción básica que es posible dirigir a concepciones similares: en verdad, aquel presupuesto necesario no es más que el resultado de la adopción de un punto de vista teórico sobre el derecho convertido en irrefutable mediante una decisión definitoria, al que se le otorga el privilegio de constituir la única vía de acceso al conocimiento jurídico. De esta manera, toda alternativa queda de antemano eliminada. ${ }^{30}$

Como esta tesis sobre el conocimiento jurídico formulada en la metateoría implica en realidad una teoría acerca del derecho, se siguen las siguientes consecuencias para la ciencia jurídica:

so Cf. Lakatos, ob. cit., p. 106. 
a) No "existen" normas no susceptibles de fundamentación, esto es, no susceptibles de incorporarse en una estructura sistemática determinada.

b) Por consiguiente, todo enunciado que pretenda describir o formular hipótesis acerca de normas que carezcan de esa fundamentación, carece a su vez de fundamentación, no pertenece a la ciencia jurídica y necesariamente tiene que ser excluido del discurso científico.

En la medida en que no puede descartarse a priori la posibilidad de otros enunciados o formulaciones teóricas, el significado último de ambas afirmaciones consiste en una directiva dirigida a los científicos para que actúen a tenor de la misma, esto es para que no reconozcan otras normas y para que no formulen otros enunciados. ${ }^{31}$

Pienso que en la base de esta directiva se encuentra la confusión básica que conduce a una identificación de los problemas de fundamentación. Esto es, la confusión entre el problema de fundamentar directivas o normas que atañen a la praxis jurídica o moral, y el problema de fundamentar enunciados como resultado de procesos de conocimientos; que, en todo caso, ante cualquier directiva de conducta sea posible una fundamentación es, ciertamente, una hipótesis teórica acerca de normas que tiene que ser probada en tanto pretenda un carácter cognoscitivo. A menos que se admita que es función de la ciencia actuar sobre las normas de manera de adecuarlas a un ideal racional.

Y en realidad, gran parte del pensamiento jurídico vigente acepta aquella directiva y en consecuencia acepta esta función epistemológica de la disciplina jurídica. ${ }^{32}$

31 La observación es pertinente en relación a toda teoría de la ciencia que postule la reducción de todos los enunciados de la ciencia a una única clase privilegiada. Aunque existen, obviamente, diferencias básicas entre una epistemología de base kantiana como la que presumiblemente admite Kelsen, y las tesis del empirismo de Carnap, es sintomático que no haya formulado la objeción de que, en definitiva, el postulado de que el discurso científico se reduce a un discurso formal acerca de estructuras como dato inmediato consiste en una directiva política-científica. Cf. Kambartel, ob. cit., p. 186.

32 Sobre el papel no epistemológico del postulado de "racionalidad del 
De todas formas, la denominada crisis del modelo justificacionista del conocimiento tiene que tener efectos inmediatos en el conocimiento jurídico: pone en cuestión la idea según la cual la racionalidad de la "realidad" jurídica tiene que medirse según los moldes de ese modelo. La idea alternativa que venga a sostener que el "derecho" es el resultado de decisiones cuya "validez" no depende de normas consideradas fundamentale ${ }^{33}$ no conduce necesariamente a "irracionalismos" en el terreno de la teoría, puesto que tampoco conduce necesariamente a "irracionalismos" en el terreno del derecho.

derecho, que en definitiva conduce a una modificación del derecho por parte de los juristas". Cf. Lezsek Nowak: La racionalité du législateur comme elément de l'interpretation juridique, Logique et Analyse 45 (1969), pp. 65-86.

88 Luhmann, ob cit., p. 105. 
The recent discussion about the foundations of scientific knowledge has affected the social sciences and the knowledge of law. The traditional answers, those of the empiricist and of the rational intuitionists, have been questioned and we could talk of a "crisis of the justificationist model".

In the foundation of juridical knowledge there are two basic questions: Are there basic statements in the legal science? If so, how are they justified? However, most of the proposed solutions refer not to these questions, but rather to the problem of the normative foundations of the law as a system of norms. In fact, Kelsen's "basic norm" seems to be a foundation both of the law and of its knowledge, which creates a typical source of confusion.

Two alternatives are analyzed in this article: Are the statements that constitute the foundations norms or are they theoretical statements? According to the first, legal scientists formulate normative statements and not theoretical ones. The question is, then, how to justify the basic norms, whether by reason alone or with empirical help. According to the second, theoretical statements can justify the validity of the norms. If that is not possible, then the only thing that can be done is to justify the knowledge of the law, but not the law itself.

Another interpretation would be to consider both justifications as parallel though not as identical, taking the basic statements of the legal science as corresponding to the basic legal norms. The normative language about the norms would be isomorphic. According to this view, the unity of legal science is a "reflex" of the systematic character of the legal "reality". After pointing out several objections to this view, the author considers a possible solution, offered by Kelsen's neo-Kantian version, that holds that the conceptual apparatus used to describe the legal norms is an epistemological category necessary for the knowledge of legal reality. The author carefully analyzes the consequences of this thesis and concludes that it is only a rationalist version of 'justificationism', open to the standard objections made to all models of this sort.

[Javier Esquivel] 